\title{
Assessment of Sanitation Practices and Attitudes of The Kotoko Community in Suame, Kumasi, Ghana
}

\author{
Roland S Kabange* \\ Department of Civil Engineering, Kumasi Technical University, Ghana
}

*Corresponding author: Roland S Kabange, Department of Civil Engineering, Faculty of Engineering and Technology, Kumasi Technical University, Kumasi, Ghana.

Received Date: December 15, 2018

Published Date: March 22, 2019

\begin{abstract}
Dangerous and unhealthy sanitation practices, attitudes, and beliefs significantly contribute to adverse public health outcomes in low-income peri-urban communities of developing countries. This research explores sanitation practices and attitudes of the Kotoko community in Kumasi (Ghana) through household survey triangulated by in-depth and key-informant interviews and transect walks. In 67 households lived 2,226 people with average household and family sizes of 33 and four respectively. The 'sanitation facilities' used in the research community were broadly grouped into community, private, and open (or inappropriate) defecation. The results showed that two-thirds of respondents reportedly defecated in the community pour-flush (PF) latrine, while $9 \%$ of respondents were open defecators, and the rest defecated in private (either own or neighbor's). Among the two-thirds of respondents who used the community PF latrine, 56\% (a larger majority men) were dissatisfied with its condition on grounds of poor maintenance and queuing, while $8 \%$ attributed the dissatisfaction to the distance of the facility from their homes (proximity concerns). These results also provided an initial indication for community sanitation improvement support. The majority (83\%) of the open defecators were men and all were willing to participate in community sanitation projects. The research community sanitation expenditure was categorized into low ( 0 - Ghø4.9), medium (Ghø5.0 - Ghø9.9), and high (Ghø10.0 - Ghø14.9) payment bands and $61 \%, 29 \%$, and $10 \%$ of respondents respectively paid within these bands - giving an average expenditure per capita per month as Ghø4.95 (USD3.01). There was no evidence to suggest that either willingness to pay and use a sanitation facility was age-sensitive, or willingness to participate in community sanitation projects was gender-sensitive in the research community when Pearson chi-square $(\chi 2)$ non-parametric tests were conducted. Regular facility cleaning (15\%) and pit emptying (15\%) were significant reported operation and maintenance practices at the community facility. The research findings indicated potential overuse and poor maintenance of the community PF latrine, suggesting inadequate sanitation provision. There was however a high sense of community participation spirit in sanitation projects. Improved maintenance of the community PF latrine, and either the construction of an additional latrine or expansion of the existing one are recommended.
\end{abstract}

Keywords: Sanitation practices and attitudes; Peri-urban; Latrine usage; Community latrine; Ghana

\section{Introduction}

Cairncross \& Satterthwaite [1] contend that adequate sanitation would reduce the spread of diseases and lead to wider social, economic and environmental benefits. The global challenge of improved sanitation access is compounded by an existing gap between attitude, practice, and knowledge [2]. Dangerous practices, attitudes and beliefs, poverty, poor governance, extreme climate, and high population densities are found to increase the likelihood of negative public health outcomes [3]. A research on women sanitation practices in Kenya found that they defecated in plastic bags and threw them on streets because they did not want to be seen using sanitation facilities often [4]. The impact of unhygienic practices on the health of communities is devastating. The unhealthy open defecation practice, for instance, degenerates into deadly diseases such as tuberculosis, cholera, hepatitis, dysentery, typhoid and diarrhoea [5]. The author argues that Ghana is currently practicing nearly $100 \%$ open defecation in principle, as about 5.2 million Ghanaians (or $19 \%$ of total population) openly defecate [6], while collected and stored excreta and other waste are mostly improperly disposed, which technically, equates to open defecation. The repercussions of open defecation and inappropriate waste disposal are eutrophication of aquatic life, adverse health 
implications on Ghanaians, and environmental degradation. Water, sanitation, and hygiene (WASH) interventions in schools are however found to improve behavioural practices and enhance knowledge in developing and developed countries alike [2], but these intervention outcomes cannot be realized without adequate investment in the sanitation subsector.

A South African study found that sanitation investment can lead to the adoption of safer and hygienic practices, with accrued benefits - morbidity and mortality reduction, life expectancy and general health improvement, and healthcare cost savings [7]. Unhygienic sanitation practices such as indiscriminate excreta, solid, and liquid waste disposal adversely affects individuals, families, and economies in terms of life quality, education, and development, promotes diseases and poor health [8]. Morgan [9] argues that poor health in turn places individuals and families in a cycle of poverty and lost income. Over 50 communicable diseases are associated with poor sanitation practices and attitudes, often resulting in millions of premature deaths annually, especially children [10] Health education and promotion strategies to ensure households understand and practice healthy waste management to prevent diseases are therefore necessary [2]. Socio-cultural preferences, which are a function of sanitation practices and attitudes, play a significant role in sanitation facilities selection and usage [11], which lead to sanitation improvement and ultimately improved health. For instance, while it is expected that sanitation facilities users would prefer to sit rather than squat to defecate, recent research found that users in a predominantly Muslim community in Ghana preferred to sit in a specific direction (North-South) during defecation so as not to face Mecca nor give their back to it $[12,13]$.

\section{Research Aim and Objectives}

There currently exist dangerous and unhealthy sanitation practices, attitudes, and beliefs in Ghana that significantly contribute to adverse public health outcomes. Adverse health outcomes in turn put individuals and families in a cycle of poverty, and increased healthcare cost to economies. This research aim is therefore to assess the sanitation practices and attitudes in the low-income high-density peri-urban Kotoko community to inform future sanitation interventions. Based on the research aim, the following under-listed objectives are therefore set to:

- Determine the research community's initial and current sanitation situations, including its history and initial prevalent common diseases;

- Determine the research community's sanitation practices, attitudes, and beliefs; and

- Make recommendations to inform future sanitation improvement interventions.

\section{Kumasi and the Research Community (Kotoko)}

Kumasi is Ghana's second largest city and capital of the most populous region (Ashanti). Kumasi's population is roughly 1.6 million with a land area of $22,300 \mathrm{~km}^{2}$, residents are predominantly
Christians (79\%) and Muslims (16\%), 1\% belongs to other religious denominations, and 4\% reported no religious affiliation [14]. English and languages of the Akan tradition are the main languages spoken in Kumasi. Four main streams (Daban, Sisa, Wiwa and Subin) flow through the city, which then join the Oda River downstream. Characteristic of Kumasi's drainage system is a concrete drain superimposed on the Subin to avert flooding in the city. This concrete drain, however, has now been turned into a "solid and liquid waste super-highway" due to the dumping of all sorts of wastes in it. Figure 1 shows the map of Kumasi, the research community (Kotoko), other Sub-Metropolitan areas, and major roads.

Kotoko is a multi-ethnic predominantly Muslim community in the Suame district and located close to the Kumasi City Centre (Kejetia). Households are built mainly from mud and bamboo, and roofed using old rusted corrugated iron sheets. The community is a heterogeneous one and composed of descendants of immigrants from northern Ghana. It is a high-density slum community characterized by inadequate infrastructure, land tenure challenges, and a mix of high- and low-income areas. Although predominantly poor, the socio-economic profile of the community is mixed (Figure 1).



Figure 1: Map of Kumasi depicting Sub-Metro areas, research community (Kotoko), and major roads Source: Maoulidi (2010).

\section{Survey Methodology and Ethics}

Three peri-urban communities (Kotoko, Akwatia Line, and Race Course) in Kumasi were preliminarily short-listed for the research based on the defining elements of a peri-urban community - infrastructure situation, population density, estimated level of community co-operation, and household income. Kotoko community in Suame (Kumasi) was finally selected for the research on the strength of the defining elements. Community meetings were held at the elders, unit committee, and community levels before the household survey to brief them on the research and gain the community's informed-consent and willingness to participate. In compliance with Muslim tradition and norms, financial tokens were provided after the elders' meeting and key-informant interview. Contact was established with key stakeholders (Kumasi Metropolitan Assembly (KMA), Kumasi Technical University 
(KsTU), and The University of Leeds) at the beginning of the household survey. Though there are currently no ethics standards for general research in Ghana, ethical approval was sought from The University of Leeds for the household survey. Appropriate permissions were also obtained from KMA and the community, and attempts were made to phrase questions in a culturally acceptable manner. A local translator was available throughout the fieldwork, as most community members had neither formal education nor common language [15].

\section{Sampling and questionnaire design}

The questionnaire was designed, pre-tested, and customized to suit local circumstances. The pre-test was to identify and rectify flaws in the questionnaire design, unearth its positive features, determine interviews timelines, and any reviews required to make the questionnaire self-explanatory, accurate and reliable. The pretest commenced on a Sunday at $6 \mathrm{am}$, as it was the most convenient day and time to get household members at home. A total of ten randomly-selected respondents was interviewed in the pre-test. Due to hot temperatures of about $40{ }^{\circ} \mathrm{C}$, the pre-test was limited to the morning session only. Sixty-seven respondents overall were interviewed in the household survey, one from each household. One household member (household head, family head or an adult) of at least 16 years was randomly identified and interviewed. The identified respondent was briefed on the interview process and requested to either complete the questionnaire if educated, or translation was done, and the respondent's exact answers were written down. When a potential respondent refused to offer an interview, the next nearest adult member of the household was approached, and the process was repeated. To avoid interviewing households twice, a mark was left at the entrance of households interviewed before proceeding to the next. Completed questionnaire were reviewed at the end of each interview day to improve data collection quality. Three consecutive Sundays were used to complete the questionnaire administration. The household survey questionnaire consisted of six sections and provided information on socio-demographic and housing characteristics, water supply and excreta disposal, community participation in sanitation projects, existing sanitation provision, operation and maintenance, and sanitation expenditure.

To gather qualitative data on the community, observation and unstructured interviews were additionally employed. Observation took the form of a transect walk to gather information on behaviour, environmental conditions, physical structures, water use patterns and practices, and defecation practices, attitudes, and beliefs. Unstructured in-depth interviews with two community elders were also conducted. The community history, experiences, and situations were documented and transcribed. The two interviews took place on two separate occasions in the elders' homes and lasted about 30 minutes per interview. An interpreter service was provided who directly translated the informants' perspectives as expressed in their own words. The gathered information was triangulated by two key-informants' interviews where necessary to minimize bias, errors, and increase findings reliability. UNDP [16] and UN MDG [17] publications on household survey design were useful methodology material.

\section{Data analysis}

The analysis was primarily based on primary data gathered from various methods - household questionnaire, field observation, key-informant interviews, transect walk, while peer-reviewed published literature was the secondary data used. Use of multiple methods ensured data triangulation by allowing for cross-checking of information to validate the results and conclusions arrived in the research. A mix of qualitative and quantitative approaches was applied to explore gathered data. Collected quantitative data were then coded and analyzed using Statistical Package for Social Scientists (SPSS). Tabulation, cross-tabulation, graphs and charts were used to analyse data for trends and patterns. Qualitative data analysis approaches such as documentation, coding, and categorization were however used for qualitative data. Filtering was applied to analyse specific selected subset of data, as opposed to cross-tabulation that compared pairs of questions. Where two variables were likely to show association, Pearson non-parametric chi-squared test was conducted at 95\% significance level.

\section{Result and Discussion}

A family in this research referred to a person (or persons) who lived together in a structure (or part of it) and were catered for as a unit with the same house-keeping arrangement. A household, however, referred to single or multiple families who lived in a single compound structure. A worker was anyone who did any form of activity that directly or indirectly brought financial rewards to support the family. With a total of 67 households, the average household size was 33, and household sizes ranged from 3 - 113. These results were in sharp contrast to an average household membership of $4-6$ people when sanitation attitudes and practices study was conducted in Kenya [2]. An earlier work in Kumasi (Ghana), however, found that over $55 \%$ of households lived in buildings with more than 10 people [18]. The average household size of 33 was comparable to MICS [19] data that found $29 \%$ and $28 \%$ of 6,302 sampled households in Ghana had sizes of 45 and 2-3 members respectively. The research community had 543 families with an average family size of four. Huge variations therefore existed in household and family sizes in the research community.

\section{Household survey and demographic data}

The research community population distribution was skewed in favour of the adult category (summarized in Table 1). From a total of 2,226 inhabitants, children five years and under constituted the least proportion (15\%) of the population, 57\% were adults 18 years and above, and people between 5-8 years constituted $28 \%$ of the community population. A significant $42 \%$ of the community were workers. Most (66\%) respondents were men, and 70\% were household heads or family heads, $30 \%$ of whom were women. Mariwah \& Drangert [20] argued that most often the heads of household were men, and most women expected husbands (or 
men) to discuss matters related to the household, which explained why more men than women were interviewed. On the contrary, however, a sanitation practices, attitudes, and knowledge research conducted in Western Kenya found that the majority $(87.5 \%)$ of respondents were women aged between 21 and 30 [2]. The same research further explained that men opted for women to be interviewed because of gender roles. An interesting finding that linked age to sanitation facility choice showed that people were likely to opt for an improved sanitation facility as they grew older [21] (Table 1).

Table 1: Research Community Demographics.

\begin{tabular}{|c|c|}
\hline Age Band (years) & Percentage of Population \\
\hline $0-5$ & $15 \%(323)$ \\
\hline $6-18$ & $28 \%(629)$ \\
\hline Research community total population & $57 \%(1,274)$ \\
\hline \multicolumn{2}{|c|}{ Household Parameters } \\
\hline \multicolumn{2}{|c|}{ Largest household size } \\
\hline \multicolumn{2}{|c|}{ Average household size } \\
\hline Least household size \\
\hline Research community total number of \\
households \\
\hline \multicolumn{2}{|c|}{ Family Parameters } \\
\hline Largest number of families in household \\
\hline Average number of families in household \\
\hline Least number of families in household \\
\hline $\begin{array}{c}\text { Total number of families in research } \\
\text { community }\end{array}$ \\
\hline
\end{tabular}

Note: The figures in parentheses are the community's population within the age bands.

\section{Research community history}

Kotoko was founded around 1949 by Nazindin Gumah, and the first chief was Ashanti King Prempeh I. Nazindin Gumah once went hunting and got a porcupine under a big tree, which was sent to the Ashanti King in accordance with tradition. The king then named the place where the porcupine was found "Kotoko", traditionally meaning "the porcupine". Kotoko's development therefore started with the settlement of Nazindin Gumah around the big tree where the porcupine was found. The community expanded from an initial population of 90 to the current size of 2,226. Rent at the time was either very cheap or non-existent and was paid directly to the Ashanti King. Community members were predominantly butchers and hunters while farming was less commonly practiced.

The first and only sanitation facilities in 1949 and early 1950s were two unlined pit latrines without superstructures. Domestic solid waste was dumped directly into the nearest bush. Four streams existed as water sources for the research community at the timePinchem, Tokoro, Ankora, and Kotoko. Kotoko is therefore currently not only the name of the research community and a stream, but also the name of an Ashanti football club in Kumasi. While the first two streams were strictly reserved as drinking water sources, Ankora and Kotoko were used for all other purposes. There was initially no school in the community or its surroundings. The only religion then was Islam, and in the early 1950s Alhaji Mohammed Waala built a "makaarata" (a special school for Muslims) located behind New Bantama but near Alang Construction Limited where Muslim teachings were delivered.

Common diseases that reportedly existed in the community were measles, asthma, chicken pox, and body-related health condition such as boils. A respondent however remarked that:

"There was absolute peace and happiness notwithstanding the abject poverty and disease that prevailed then."

Another respondent stated that:

"Though sanitation and water supply facilities were highly unhygienic, and illiteracy and poverty levels were very high compared to the present, community members had comparatively better health, lived peacefully and had the love for one another."

Medical facilities at that time were unavailable, and the community resorted to traditional treatment methods as follows:

- Herbs called "Egro" were used to treat asthma - it is boiled, squeezed and the fluid drunk and administered to the body;

- Measles were treated by applying "akpeteshie" (a local gin) to the body; and

- Chicken pox was treated using soaked "Lelley" (leaves of a common tree) for bathing and drinking.

\section{Research community sanitation situation}

The research community sanitation provision consisted mainly of a 14-seater pour-flush (PF) community latrine with separate gender arrangements - seven seats each for males and females. There were a few private sanitation facilities in the high-income housing located along the community's boundaries. The PF latrine consisted of three main components - a separate superstructure which houses the 14 squat holes and an integrated water seal linked by an interconnected PVC pipe to a septic tank. It also incorporated a ventilation pipe and a flyscreen on the septic tank whose functions were to control odour and flies. The few private facilities were mainly PF to individual household septic tanks. The research community was predominantly Muslim and anal cleansing with water was largely the norm. Though the facility was initially designed for self-flushing from an overhead water tank, it was converted to a PF facility because the automatic-flush mechanism broke down years ago had not been repaired since. Attached to the facility superstructure were four bathrooms - two for females and two for males. Under the Government of Ghana sanitation policy, the PF latrine was managed by the community's Unit Committee with oversight supervision by the Assemblyman. The facility's operation and maintenance $(0 \& M)$ costs were covered by the generated revenue under the pay-per-use policy.

\section{Reported water supply situation}

The household survey conducted found that the reported water sources ranged from drainage canals to wells. The community's 
two main water sources were: wells - 64\% (43) of respondents; and vendors - $27 \%$ (18) of respondents, compared to a reported $38 \%$ of Ghanaian households who used unprotected water sources (such as springs, rivers and open wells) for drinking and cooking [22]. A review found that $45 \%$ of rural households in Sub-Saharan Africa used wells as their main water sources [23]. Among the 43 respondents who cited wells as their main water sources, nearly all (92\%) admitted to no treatment before drinking. Out of the 18 respondents who sourced vendors water, $79 \%$ did not treat it before drinking. Mittal et al. [24] had an identical result when they found that $78 \%$ of respondents in a sanitation attitude and practices study in Tamil (India) did not treat water before drinking. Boiling, chlorination, and mineral water use were rarely practiced in the community. This result concurs with published data on water treatment in Ghana [19] which showed that about 92\% of Ghana's population did not treat their drinking water and drinking water treatment by households was about $3 \%$.

\section{Types of sanitation facilities used}

Sanitation facilities used in the research community were broadly grouped into three categories - community, private (own or relatives'), and open (or inappropriate) defecation. Compound sanitation facilities (popularly known as household toilets), though common in Ghana [25], were not used by the research community. Figure 2 shows that two-thirds (67\%) of respondents reportedly defecated in the community latrine, while under 1 in 4 (24\%) used private facilities. Open defecation was practiced by $9 \%$, compared to $19 \%$ open defecation in Ghana $[6,26]$. Though the $9 \%$ open defecation might be small, it was a significant fraction, especially if this could be translated to the whole community. Knowledge that $9 \%$ of respondents defecated openly provided an initial indication for sanitation improvement support in the community. The lowincome non-sewered research community therefore had poor sanitation provision, and most people used the shared community PF latrine (Figure 2).

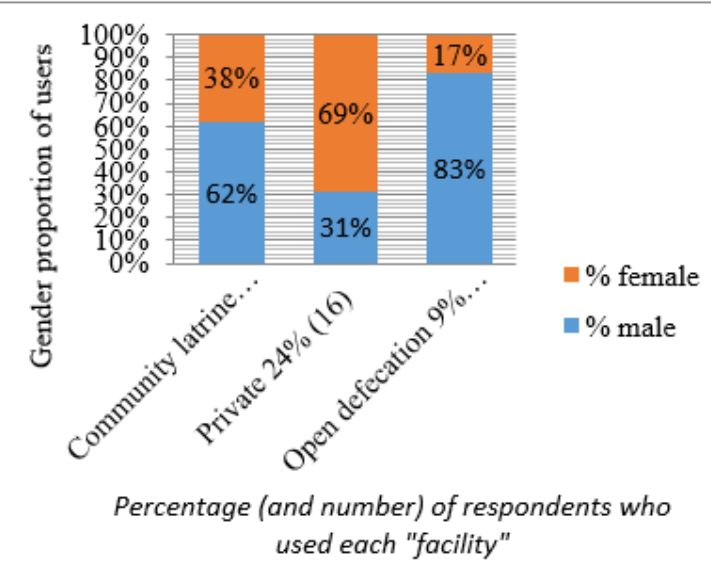

Figure 2: Proportion of respondents who used each type of "facility" by gender.

Users of community pour-flush (PF) latrine: Research shows that sanitation facilities' dirty conditions were likely deterrent to users [27]. Among the $67 \%$ of respondents who used the community PF latrine, 56\% were dissatisfied with its condition, while most (88\%) private facility users were satisfied (Figure 3). Further investigation revealed that community latrine users and open defecators views were considered more critical in the selection and design of new and improved sanitation systems for the community. Most (68\%) of the dissatisfied community PF latrine users were men. Most respondents reported queuing and poor maintenance, while handwashing and proximity to sanitation facility were only minor concerns for dissatisfaction (Figure 4). Whereas $60 \%$ of the community PF latrine users reported good or very good hygienic conditions, two-fifth (40\%) reported it was in bad or very bad state. The dominant problems cited by about $80 \%$ of respondents were insect nuisance, smell, and facility not emptied. Most (96\%) users were willing to participate in community sanitation projects, and close to $40 \%$ reported disease affected the community due to poor sanitation - suggesting a high sense of community participation spirit in sanitation projects, and the need for sanitation improvement.



Figure 3: Satisfaction levels among users of each type of "facility".

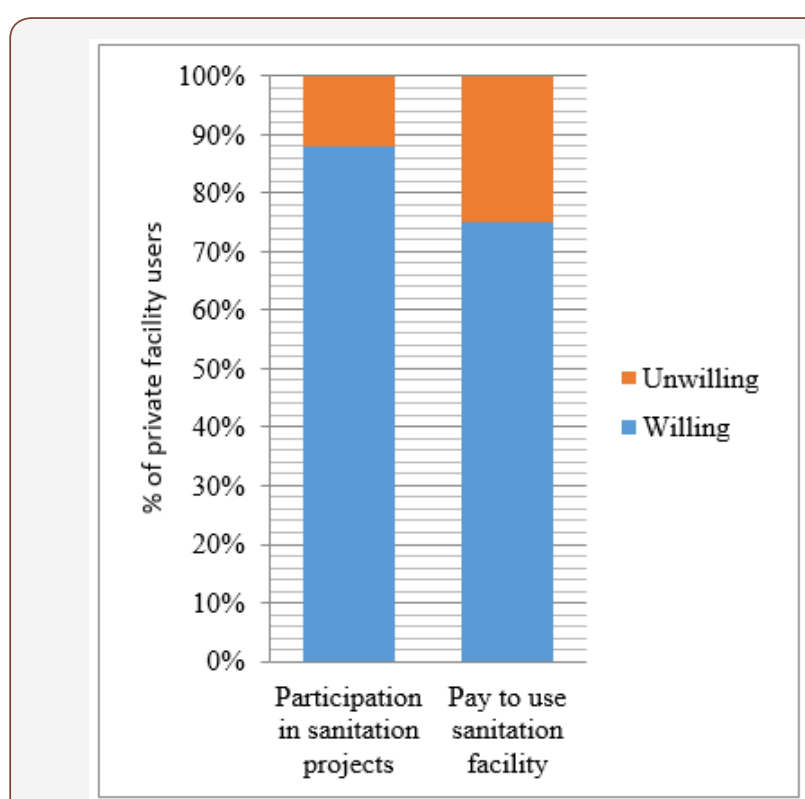

Figure 4: Private facilities users' levels of willingness". 
Users of private facilities: Out of the $24 \%$ of respondents who defecated in private facilities, $81 \%$ used their own facilities and the rest defecated in relatives', and all reported a good or very good hygienic state of the facilities they used. In contrast to community PF latrine, the data suggested that private facilities had comparatively better hygienic conditions and were better operated and maintained. Figure 4 demonstrated that despite ownership of their own sanitation facilities, users of private facilities reported impressive willingness to participate in community sanitation projects, and to pay and use sanitation facilities.

Open (or inappropriate) defecators: Out of the $9 \%$ of respondents who reported practicing open defecation, the majority (83\%) were men and all were willing to participate in community sanitation projects. The spirit of community participation was high within the research community. Literature, however, shows that enthusiasm for participation does not often necessarily translate into results in practice [28].

\section{Research community sanitation provision}

Studies show that increasing distance to sanitation facilities is associated with decreased facilities use and increased open defecation, and dirty conditions are likely sources of dissatisfaction and deterrent in using the facilities [27]. Among respondents dissatisfied with their sanitation facilities, $48 \%$ suggested the construction of another facility to complement the already existing one. However, among the dissatisfied respondents who used the PF community latrine, $28 \%$ reported queuing at the facility as source of dissatisfaction, and about $8 \%$ attributed such dissatisfaction to the distance of the facility from their homes. These findings suggest unsatisfactory sanitation provision in the research community, and raised concerns of accessibility, convenience and comfort - factors that could de-motivate users. The findings also showed that 2 in 3 respondents defecated in the community facility. Given the community's 2,226 population (Table 1 ), about 1,500 people (excludes those who practice open defecation) in the community used the only PF community facility. There was therefore an indication of potential overuse of the facility, which again suggested inadequate sanitation provision concerns.

\section{Age and gender perspectives in reported sanitation practices}

Data analysis by age and gender in sanitation projects is important because women and men at various stages of their lives tend to behave differently towards sanitation and hygiene issues in real life [29]. Research in Kenya, for instance, revealed that women defecated in plastic bags and threw them onto the streets, so they were not seen to be using latrines too often [4]. Age and gender are therefore compared with relevant variables and the trends further analyzed.

The age perspective: Respondents' ages were analyzed against willingness to pay to use a sanitation facility: $30 \%$ of respondents aged 15-45 were unwilling to pay compared to $17 \%$ aged $46+$. of the $30 \%$ respondents aged $15-45$ unwilling to pay to use a sanitation facility, the majority (73\%) used the community latrine and only three used private facilities. This would suggest that despite the lack of willingness to pay, they occasionally paid to use a facility. Pearson chi-square $\left(\chi^{2}\right)$ non-parametric test for association between age and willingness to pay to use sanitation facility was conducted with the null hypothesis $\left(\mathrm{H}_{0}\right)$ that 'willingness to pay to use a facility does not depend on age of respondents. The calculated $\chi^{2}$ value was 1.61 and the critical value of $\chi_{(1)}^{2}(5 \%)=3.84$. As the calculated $\chi^{2}=1.61<3.84$, we accept the null hypothesis and reject the alternative. There was no evidence to suggest that willingness to pay to use a sanitation facility was age-sensitive.

The gender perspective: Comparison of respondents' gender to willingness to participate in community sanitation projects showed that out of the 62 respondents willing to participate, $34 \%$ were women. Pearson chi-square $\left(\chi^{2}\right)$ non-parametric significance test for association between gender and willingness to participate was conducted with the null hypothesis $\left(\mathrm{H}_{0}\right)$ that 'willingness to participate in community sanitation projects does not depend on the gender of respondents. The calculated $\chi^{2}$ value was 2.6 and the critical value of $\chi_{(2)}^{2}(5 \%)=6.0$. Since the calculated $\chi^{2}=2.6<6.0$, we accept the null hypothesis and reject the alternative. There was therefore no statistical association between gender and willingness to participate in community sanitation projects. Respondents' defecation places were also compared with their gender. Pearson chi-square $\left(\chi^{2}\right)$ non-parametric test for independence was used to investigate the relationship between gender and defecation places of respondents with the null hypothesis $\left(\mathrm{H}_{0}\right)$ that 'place of defecation of respondents in the research community does not depend on gender. The calculated $\chi^{2}$ value was 1.1 and the critical value of $\chi_{(2)}^{2}(5 \%)=6.0$. As the calculated $\chi^{2}=1.1<6.0$ (critical value), we thus accept the null hypothesis and reject the alternative. We therefore concluded that there was no evidence that willingness to participate in community sanitation projects in the research community was gender-sensitive, nor was respondents' place of defecation gender-sensitive.

\section{Reported expenditure on sanitation}

Categorized into low (0 - Gh\$4.9), medium (Gh\$5.0 - Gh\$9.9), and high (Gh\$10.0 - Gh\$14.9) payment bands, respondents' expenditure per month on sanitation was computed. Across the payment bands, $61 \%, 29 \%$ and $10 \%$ of respondents paid within the low, medium and high payment bands respectively on sanitation (Table 2) which translated to Ghø4.95 (USD3.01) average expenditure paid per person per month on sanitation. This monthly expenditure of USD3.01 on sanitation per capita was about 24 times higher than the USD1.50 paid annually per capita on sanitation services in Kumasi 24 year ago when a similar research was conducted [22]. While this current research did not investigate the reasons that accounted for this vast sanitation expenditure disparity, it was likely that inflation over the period played a role. The results showed that four, two and none of the respondents among the low, medium, and high payment bands respectively defecated inappropriately. Table 2 showed that some respondents who 
reported spending on sanitation practiced open defecation. It was likely that this group used the sanitation facility only occasionally for some reason and might prefer to identify with open defecators. The data also showed that 4 in $5(80 \%)$ respondents in the high payment band used private facilities, and the rest (20\%) used the community PF latrine. Over two-thirds (67\%) of respondents who defecated in the community latrine reported paying the least (low payment band), while $30 \%$ paid within the medium payment band (Table 2).

Table 2: Respondents' Sanitation Expenditure Across Three Payment Bands.

\begin{tabular}{|c|c|c|c|}
\hline Payment Band & $\begin{array}{c}\text { Payment/ } \\
\text { Month/Family } \\
\text { (Ghథ) }\end{array}$ & $\begin{array}{c}\text { Proportion of } \\
\text { Respondents } \\
\text { (\%) }\end{array}$ & $\begin{array}{c}\text { Proportion } \\
\text { of Open } \\
\text { Defecation (\%) }\end{array}$ \\
\hline Low & $0-4.9$ & 61 & 8 \\
\hline Medium & $5.0-9.9$ & 29 & 4 \\
\hline High & $10.0-14.9$ & 10 & 0 \\
\hline
\end{tabular}

\section{Operation and maintenance concerns}

The most important operation to ensure effective sanitation is to keep latrines clean and may have negative health effects if they are not properly cleaned and used [30]. When maintenance data was analyzed, it was found that out of $70 \%$ of respondents who reported infrequent maintenance, $38 \%$ of them were not satisfied with the facility's condition, and $62 \%$ were satisfied. Therefore, $27 \%$ of respondents reported infrequent maintenance and were also not satisfied with the condition of the sanitation facility. The reasons reported by this fraction of respondents were mixed: $28 \%$ of them cited queuing at the facility; $11 \%$ mentioned the distance of the sanitation facility from home; $22 \%$ reported unbearable heat and odour; $28 \%$ said the facility was not well kept; and the absence of handwashing facilities was a minor concern.

Keeping latrines clean limits human contact with excreta, reduces flies and smell inside latrine, and improves its appearance, which promote latrine use [31]. Mixed opinions were reported when operational problems of the PF facility were compared with respondents' level of satisfaction. Though $60 \%$ of respondents were reportedly satisfied with the facility's condition, under half $(48 \%)$ reported operational problems, and more than half (52\%) had no operational concerns. Among respondents who were satisfied but reported operational problems, most (95\%) cited insect nuisance, foul smell, facility not being emptied, and a combination of these as their concerns. Insect and foul smell accounted for over half (56\%) of these concerns. Respondents' views seemed to suggest that the community PF latrine was poorly maintained.

\section{The way forward}

The household survey collected some initial data on respondents' suggestions for improvement, and concerns with the current PF sanitation facility. It was found that nearly half (48\%) of respondents were reportedly dissatisfied with the sanitation facility (shown in Figure 5) and suggested the construction of another facility to complement the existing one to improve the sanitation situation. Regular cleaning of the facility (15\%), and regular pit emptying (15\%) were also reported by a smaller proportion. The provision of refuse containers and upgrade of the existing PF facility to a water closet (WC) was cited by a minor proportion. Out of the $48 \%$ dissatisfied respondents who suggested the construction of another facility, queuing was the concern of nearly half (46\%), while the rest reported unbearable heat, odour, and poor maintenance (Figure 5). This current research did not investigate the reasons that accounted for this vast sanitation expenditure disparity, it was likely that inflation over the period played a role. The results showed that four, two and none of the respondents among the low, medium, and high payment bands respectively defecated inappropriately.

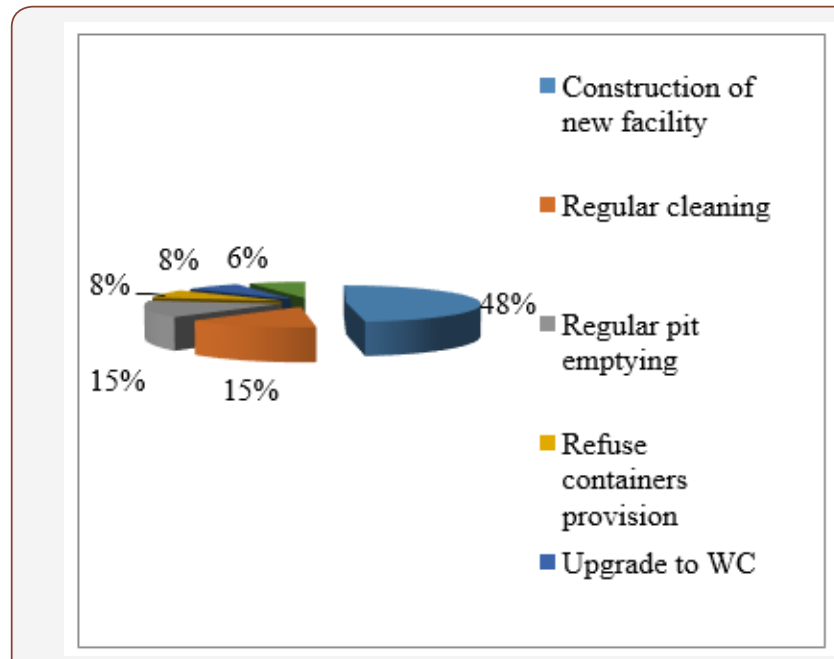

Figure 5: : Reported ways of improvement.

\section{Conclusion}

Despite several interventions to reverse the trend, unhealthy and dangerous sanitation practices, attitudes, and beliefs significantly contribute to the negative public health situation in most developing countries. This research undertaken in lowincome peri-urban Kotoko community located in Kumasi (Ghana) sought to assess the sanitation practices and attitudes to inform future sanitation interventions. In 67 households lived 2,226 people with average household and family sizes of 33 and four respectively. The community reported water supply sources were wells and vendors, and water was not treated before drinking. The 'sanitation facilities' used in the research community were broadly grouped into community, private, and open (or inappropriate) defecation. The results showed that two-thirds of respondents reportedly defecated in the community PF latrine, while 9\% of respondents were open defecators - a result that provided an initial indication of the need for community sanitation improvement. Among the two-thirds of respondents who used the community PF latrine, $56 \%$ (a larger majority men) were dissatisfied with its condition on grounds of poor maintenance and queuing, and $8 \%$ attributed the dissatisfaction to the distance of the facility from their homes. Overall, the results showed inadequate sanitation provision, and overuse and poor maintenance of the community PF latrine. The majority (83\%) of the open defecators were men and all were willing to participate in community sanitation projects highlighting the high sense of community participation spirit in sanitation projects. 
Nearly half $(48 \%)$ of the respondents who reported dissatisfied with their sanitation facility suggested new facility construction to augment the existing facility. Regular facility cleaning (15\%) and pit emptying (15\%) were other significant reported suggestions. The research findings indicated that the research community (Kotoko) $\mathrm{PF}$ latrine was poorly maintained. There was no evidence to suggest that either willingness to pay and use a sanitation facility was agesensitive, or respondents' willingness to participate in community projects and respondents' defecation places were gender-sensitive when Pearson chi-square $\left(\chi^{2}\right)$ non-parametric tests were conducted. The research community sanitation expenditure was categorized into low (0 - Ghø4.9), medium (Ghø5.0 - Ghø9.9), and high (Gh $\varnothing 10.0$ - Ghø14.9) payment bands and 61\%, 29\%, and $10 \%$ of respondents respectively paid within these bands - which translated to Gh\&4.95 (USD3.01) average expenditure per capita per month.

\section{Research Limitations and Further Work}

Cost data was limited because reliable information on operating costs of existing sanitation facilities were difficult to get. Either information was unavailable, or people were unwilling to give them. Lack of adequate government engagement meant that some of the solutions might not be institutionally viable even if technically attractive. Accurate data collection on sensitive issues presents special challenges in research. For example, acquiring individual or even household-level responses in crowded urban areas was challenging due to inevitable information leakage between respondents. Representativeness was also difficult to achieve in the research community where households' sizes ranged from 3-113. An improved representativeness could have been achieved by sampling the number of respondents in proportion to household size.

\section{Acknowledgment}

RS Kabange thanks Mrs. Esther Kabange (my wife) for her understanding and unconditional support, and The Almighty God for that special Grace, health, and protection throughout the research process.

\section{Conflict of Interest}

No conflict of interest.

\section{References}

1. Cairncross S, Satterthwaite D (1990) The Poor Die Young: Housing and Health in Third World Cities, Earthscan Publications, London, UK.

2. Wasonga J, Olang'o CO, Kioli F (2014) Improved household knowledge and attitudes on water, sanitation, and hygiene practices through school health programme in Nyakach. Kisumu county in Western Kenya, Journal of Anthropology, 6 pages.

3. WHO (2006) World Health Organization Guidelines for the Safe Use of Wastewater, Excreta and Greywater, Volume IV: Excreta and Greywater Use in Agriculture, France: World Health Organization, France.

4. Pearson J, Mcphedran K (2008) A literature review of the non-health impacts of sanitation. Waterlines 27(1): 48-61.

5. Kalbermatten JM, Middleton R, Schertenleib R (1999) Household-centred environmental sanitation, Swiss Federal Institute for Environmenta Science and Technology Ueberlandstrasse 133, Switzerland: CH-8600 Deubendorf, Switzerland.
6. WHO/UNICEF (2017) Progress on Drinking Water, Sanitation and Hygiene: 2017 Update and SDG Baselines, Geneva: United Nations.

7. DWAF (2001) White Paper on Basic Household Sanitation, Government Printers: Pretoria.

8. Phaswana-Mafuya N, Shukla N (2005) Factors that could motivate people to adopt safe hygienic practices in the Eastern Cape Province, South Africa, African Health Sciences 5 (1): 21-28.

9. Morgan J (2001) Environmental Health Promotion Capacity Building: A Training Guide Based on CARE'S Hygiene Promotion Manual, WELL Task 515, London: London School of Hygiene and Tropical Medicine, London.

10. Wright AM (1997) Towards a Strategic Sanitation Approach: Improving the Sustainability of Urban Sanitation in Developing Countries, UNDPWorld Bank Water and Sanitation Program.

11. Kabange RS, Graham J (2017) Community population density variation implications on sanitation system cost - the case of Kotoko community in Suame (Kumasi), Ghana, Journal of Civil and Environmental Research 9 (2): 46-53

12. Avvannavar SM, Mani M (2007) A conceptual model of peoples approaches to sanitation. Sci Total Environ. 390(1): 1-12.

13. Kabange RS, Nkansah A (2015) Peri-urban community socio-cultural preferences for, and experts' views on, sanitation options: a case study of the Kotoko community in Suame (Kumasi), Ghana, Journal of Environmental and Earth Sciences 5(18): 28-35.

14. Millennium Cities Initiative (2008) Invest in Ghana: Focus Kumasi, Canada: Columbia University, Canada.

15. Maoulidi M (2010) A Water and Sanitation Needs Assessment for Kumasi, Ghana, MCI Social Sector Working Paper Series No. 16.

16. UNDP (2012) 2010 Ghana Millennium Development Goals Report, Accra: UNDP Ghana.

17. UN MDG (2005) The Millennium Development Goals Report 2005, New York: The United Nations, New York.

18. Whittington D, Laura DT, Choe K, Hughes JA and Swarna V (1993) Household sanitation in Kumasi, Ghana: a description of current practices, attitudes, and perceptions, World Development 21(5): 733748

19. MICS (2006) Ghana: Monitoring the Situation of Children, Women and Men, Accra

20. Mariwah S, Drangert JO (2011) Community perceptions of human excreta as fertilizer in per-urban agriculture in Ghana," Waste Management and Research 29(8): 815-822.

21. Aryitey PNA (2015) Household choice of water supply and sanitation systems: contemporary Ghanaian analysis," MPhil Thesis, Accra: University of Ghana, Ghana.

22. Whittington D, Davis J, Prokopy L, Komives K, Thorsten R, et al. (2009) How well is the demand-driven community management model for rural water supply systems doing? Evidence from Bolivia, Peru and Ghana, Water Policy 11(6).

23. Grönwall JT, Mulenga M, McGranahan G (2010) Groundwater, selfsupply and poor urban dwellers: a review with case studies of Bangalore and Lusaka, Human Settlements Working Paper Series, Water and Sanitation-26.

24. Mittal A, Rajesekar VD, Thirumal P, Murali S (2016) A cross-sectional study to determine knowledge, attitude and practice of sanitation in rural areas of Tamil Nadu, India, International Journal of Community Medicine and Public Health 3(7): 1910-1914.

25. Jenkins MW, Scott B (2007) Behavioural indicators of household decision-making and demand for sanitation and potential gains from social marketing in Ghana, Journal of Social Science and Medicine 64(12): 2427-2442

26. United Nations (2016) The Sustainable Development Goals Report 2016, New York: United Nations, New York. 
27. Biran (2010) Communal toilets in urban poverty pockets - use and user satisfaction associated with seven communal toilets in Bhopal, India, WaterAid Report.

28. Roma E, Buckley C, Jefferson B, Jeffrey P (2010) Assessing users experience of shared sanitation facilities: a case study of community ablution blocks in Durban, South Africa, Water SA, 36 (5).

29. Wijk-Sijbesma C (2001) The Best of Two Worlds? Methodology for Participatory Assessment of Community Water Services, Wageningen: IRC International Water and sanitation Centre.
30. Pickford J (1995) Low-Cost Sanitation: A Survey of Practical Experiences, London: Intermediate Technology Publication.

31. Gavin, Hockley T, Joyce S (1993) Community Sanitation Improvement and Latrine Construction Program: A Training Guide, Technical report 83, Water and Sanitation for Health project, Arlington: USA. 